Applied Mathematical Sciences, Vol. 9, 2015, no. 17, 845 - 851

HIKARI Ltd, www.m-hikari.com

http://dx.doi.org/10.12988/ams.2015.4121054

\title{
A Derivation of Two Quadratic Transformations Contiguous to that of Gauss via a Differential Equation Approach
}

\author{
S. Meethal \\ School of Mathematical and Physical Sciences \\ Central University of Kerala, Periye P.O. Dist. \\ Kasaragad 671123, Kerala State, India \\ Arjun K. Rathie \\ School of Mathematical and Physical Sciences \\ Central University of Kerala, Periye P.O. Dist. \\ Kasaragad 671123, Kerala State, India
}

\section{R. B. Paris ${ }^{1}$}

School of Computing, Engineering and Applied Mathematics

University of Abertay Dundee, Dundee DD1 1HG, UK

Copyright (C) 2015 S. Meethal, Arjun K. Rathie and R. B. Paris. This is an open access article distributed under the Creative Commons Attribution License, which permits unrestricted use, distribution, and reproduction in any medium, provided the original work is properly cited.

\begin{abstract}
The purpose of this note is to provide an alternative proof of two quadratic transformation formulas contiguous to that of Gauss using a differential equation approach.
\end{abstract}

Mathematics Subject Classification: 33C20

Keywords: Gauss hypergeometric function, quadratic transformation, hypergeometric differential equation

\footnotetext{
${ }^{1}$ Corresponding author
} 


\section{Introduction}

The quadratic transformation for the hypergeometric function ${ }_{2} F_{1}(a, b ; c ; x)$ we consider here is the one originally obtained by Gauss in the form (see, for example, [1, p. 128])

$$
(1+x)^{-2 a} F_{1}\left[\begin{array}{c}
a, b \\
2 b
\end{array} ; \frac{4 x}{(1+x)^{2}}\right]={ }_{2} F_{1}\left[\begin{array}{c}
a, a-b+\frac{1}{2} \\
b+\frac{1}{2}
\end{array} ; x^{2}\right]
$$

valid when $|x|<1$ and $\left|4 x /(1+x)^{2}\right|<1$ and provided $2 b$ is neither zero nor a negative integer. Bailey [2] re-derived this result by employing the classical Watson summation theorem for the ${ }_{3} F_{2}$ series. In the standard text of Rainville [5, p. 63], the transformation (1.1) was derived using the differential equation satisfied by ${ }_{2} F_{1}$.

In 2001, Rathie and Kim [6] established two transformation formulas contiguous to (1.1) with the help of a contiguous version of Watson's summation theorem due to Lavoie et al. These are given in the following theorem.

Theorem 1. If $|x|<1$ and $\left|4 x /(1+x)^{2}\right|<1$ then

$$
\begin{aligned}
(1+x)^{-2 a}{ }_{2} F_{1}\left[\begin{array}{c}
a, b \\
\left.2 b+1 ; \frac{4 x}{(1+x)^{2}}\right]
\end{array}\right. & ={ }_{2} F_{1}\left[\begin{array}{c}
a, a-b+\frac{1}{2} \\
b+\frac{1}{2}
\end{array} ; x^{2}\right] \\
& -\frac{2 a x}{2 b+1}{ }_{2} F_{1}\left[\begin{array}{c}
a+1, a-b+\frac{1}{2} \\
b+\frac{3}{2}
\end{array} x^{2}\right]
\end{aligned}
$$

and

$$
\begin{aligned}
(1+x)^{-2 a}{ }_{2} F_{1}\left[\begin{array}{c}
a, b \\
2 b-1
\end{array} ; \frac{4 x}{(1+x)^{2}}\right] & ={ }_{2} F_{1}\left[\begin{array}{c}
a, a-b+\frac{3}{2} \\
b-\frac{1}{2}
\end{array} x^{2}\right] \\
+ & \frac{2 a x}{2 b-1}{ }_{2} F_{1}\left[\begin{array}{c}
\left.a+1, a-b+\frac{3}{2} ; x^{2}\right] \\
b+\frac{1}{2}
\end{array}\right.
\end{aligned}
$$

provided $2 b \pm 1$ is neither zero nor a negative integer, respectively.

Here we give an alternative demonstration of the quadratic transformations (1.2) and (1.3) by adopting the differential equation approach employed by Rainville. It is worth remarking that these transformations cannot be derived completely by the hypergeometric differential equation, but that a related second-order differential equation has to be solved by the standard Frobenius method.

Before we give our alternative derivation of (1.2) and (1.3) in Section 3, we first present an outline of the arguments employed by Rainville [5, p. 63] to establish the Gauss transformation (1.1). 


\section{Derivation of (1.1) by Rainville's method}

The hypergeometric function ${ }_{2} F_{1}(a, b ; c ; z)$ satisfies the differential equation [1, p. 75], [4, Eq. (15.10.1)]

$$
z(1-z) \frac{d^{2} w}{d z^{2}}+\{c-(a+b+1) z\} \frac{d w}{d z}-a b w=0 .
$$

If we put $c=2 b$ and make the change of variable $z=4 x /(1+x)^{2}$, then equation (2.1) becomes

$$
x(1-x)(1+x)^{2} \frac{d^{2} w}{d x^{2}}+2(1+x)\left\{b-2 a x+(b-1) x^{2}\right\} \frac{d w}{d x}-4(1-x) a b w=0 .
$$

If we now put $w=(1+x)^{2 a} y$, then after some simplification we find

$$
x\left(1-x^{2}\right) \frac{d^{2} y}{d x^{2}}+2\left\{b-(2 a-b+1) x^{2}\right\} \frac{d y}{d x}-2 a x(1+2 a-2 b) y=0,
$$

of which one solution is

$$
y=(1+x)_{2}^{-2 a} F_{1}\left[\begin{array}{c}
a, b \\
2 b
\end{array} ; \frac{4 x}{(1+x)^{2}}\right] .
$$

The differential equation (2.2) is invariant under the change of variable from $x$ to $-x$. Hence, if we introduce the new independent variable $v=x^{2}$ the equation describing $y$ becomes

$$
v(1-v) \frac{d^{2} y}{d v^{2}}+\left\{b+\frac{1}{2}-\left(2 a-b+\frac{3}{2}\right) v\right\} \frac{d y}{d v}-a\left(a-b+\frac{1}{2}\right) y=0
$$

We observe that (2.2) is of the same form as the hypergeometric differential equation (2.1), which therefore has in $|v|<1$ the two solutions [4, Eq. (15.10.2)]

$$
{ }_{2} F_{1}\left[\begin{array} { c } 
{ a , a - b + \frac { 1 } { 2 } } \\
{ b + \frac { 1 } { 2 } }
\end{array} , \quad \text { and } v _ { 2 } ^ { \frac { 1 } { 2 } - b } F _ { 1 } \left[\begin{array}{c}
a-b+\frac{1}{2}, a-2 b+1 \\
\frac{3}{2}-b
\end{array} ; v .\right.\right.
$$

We observe that the differential equation (2.2) has the solution (2.3) valid in $\left|4 x /(1+x)^{2}\right|<1$, provided $2 b$ is neither zero nor a negative integer. At the same time, equation (2.2) has the solutions (2.5) with $v=x^{2}$ valid in $|x|<1$. Therefore, subject to these conditions, there exist constants $A$ and $B$ such that

$$
\begin{aligned}
(1+x)_{2}^{-2 a} F_{1}\left[\begin{array}{c}
a, b \\
\left.2 b ; \frac{4 x}{(1+x)^{2}}\right]
\end{array}\right. & =A_{2} F_{1}\left[\begin{array}{c}
a, a-b+\frac{1}{2} \\
b+\frac{1}{2}
\end{array} ; x^{2}\right] \\
& +B x_{2}^{1-2 b} F_{1}\left[\begin{array}{c}
a-b+\frac{1}{2}, a-2 b+1 \\
\frac{3}{2}-b
\end{array} ; x^{2}\right] .
\end{aligned}
$$


The left-hand side and the first member on the right-hand side of the above expression are both analytic at $x=0$, but the remaining term is not due to the presence of the factor $x^{1-2 b}$. Hence $B=0$ and by considering the terms at $x=0$ it is easily seen that $A=1$. If $2 b$ is a positive integer, the second solution may involve a logarithmic term in $x$, which again requires $B=0$. This leads to the required quadratic transformation given in (1.1).

\section{An alternative derivation of Theorem 1}

We first establish the quadratic transformation (1.2). With $c=2 b+1$ in (2.1) and the change of variable $z=4 x /(1+x)^{2}$ we obtain

$x(1-x)(1+x)^{2} \frac{d^{2} w}{d x^{2}}+(1+x)\left\{2 b+1-4 a x+2 x+(2 b-1) x^{2}\right\} \frac{d w}{d x}-4 a b(1-x) w=0$,

which has a solution $w={ }_{2} F_{1}\left(a, b ; 2 b+1 ; 4 x /(1+x)^{2}\right)$. With the further change of dependent variable $w=(1+x)^{2 a} y$, we find after some simplification

$$
x\left(1-x^{2}\right) \frac{d^{2} y}{d x^{2}}+\left\{2 b+1+2 x-(4 a-2 b+1) x^{2}\right\} \frac{d y}{d x}+2 a\{1+2(b-a) x\} y=0 .
$$

A solution of (3.1) is consequently

$$
y=(1+x)^{-2 a}{ }_{2} F_{1}\left[\begin{array}{c}
a, b \\
2 b+1
\end{array} ; \frac{4 x}{(1+x)^{2}}\right] .
$$

The differential equation (3.1) is not invariant under the change of variable $x$ to $-x$, and so we cannot reduce it to the hypergeometric equation (2.1). Inspection of (3.1) shows that the point $x=0$ is a regular singular point. Accordingly, we seek two linearly independent solutions of (3.1) by the Frobenius method and let

$$
y=x^{\lambda} \sum_{n=0}^{\infty} c_{n} x^{n} \quad\left(c_{0} \neq 0\right),
$$

where $\lambda$ is the indicial exponent. Substitution of this form for $y$ in (3.1) then leads after a little simplification to

$$
\begin{gathered}
\sum_{n=0}^{\infty} c_{n} x^{n-1}(n+\lambda)(n+\lambda+2 b)=\sum_{n=0}^{\infty} c_{n} x^{n+1}(n+\lambda+2 a)(n+\lambda+2 a-2 b) \\
-2 \sum_{n=0}^{\infty} c_{n} x^{n}(n+\lambda+a) .
\end{gathered}
$$

The coefficients of $x^{-1}$ must vanish to yield the indicial equation

$$
\lambda(\lambda+2 b)=0
$$


so that $\lambda=0$ and $\lambda=-2 b$. Equating the coefficients of $x^{n}$ for non-negative integer $n$, we obtain

$$
\begin{aligned}
c_{1} & =\frac{-2(\lambda+a)}{(1+\lambda)(1+\lambda+2 b)} c_{0}, \\
c_{n} & =\frac{\{n+\lambda+2(a-1)\}\{n+\lambda+2(a-b-1)\} c_{n-2}-2(n+\lambda+a-1) c_{n-1}}{(n+\lambda)(n+\lambda+2 b)}
\end{aligned}
$$

for $n \geq 2$.

With the choice $\lambda=0$, we have

$$
\begin{aligned}
c_{1} & =\frac{-2 a}{(2 b+1)} c_{0}, \\
c_{n} & =\frac{\{n+2(a-1)\}\{n+2(a-b-1)\} c_{n-2}-2(n+a-1) c_{n-1}}{n(n+2 b)} \quad(n \geq 2) .
\end{aligned}
$$

Solution of this three-term recurrence with the help of Mathematica generates the values given by

$$
c_{2 n}=\frac{(a)_{n}\left(a-b+\frac{1}{2}\right)_{n}}{n !\left(b+\frac{1}{2}\right)_{n}} c_{0}, \quad c_{2 n+1}=\frac{(a+1)_{n}\left(a-b+\frac{1}{2}\right)_{n}}{n !\left(b+\frac{3}{2}\right)_{n}} c_{1},
$$

the general values being established by induction. Substitution in (3.2) then yields one solution of (3.1) given by

$$
y_{1}=c_{0}\left\{{ }_{2} F_{1}\left[\begin{array}{c}
a, a-b+\frac{1}{2} \\
b+\frac{1}{2}
\end{array} ; x^{2}\right]-\frac{2 a x}{2 b+1}{ }_{2} F_{1}\left[\begin{array}{c}
a+1, a-b+\frac{1}{2} \\
b+\frac{3}{2}
\end{array} x^{2}\right]\right\}
$$

when $|x|<1$.

A second solution is obtained by taking $\lambda=-2 b$ in (3.3) to yield

$$
\begin{aligned}
c_{1} & =\frac{-2(a-2 b)}{(1-2 b)} c_{0}, \\
c_{n} & =\frac{\{n+2(a-2 b-1)\}\{n+2(a-b-1)\} c_{n-2}-2(n+a-2 b-1) c_{n-1}}{n(n-2 b)}
\end{aligned}
$$

for $n \geq 2$. This generates the values

$$
c_{2 n}=\frac{(a-2 b)_{n}\left(a-b+\frac{1}{2}\right)_{n}}{n !\left(\frac{1}{2}-b\right)_{n}} c_{0}, \quad c_{2 n+1}=\frac{(a-2 b+1)_{n}\left(a-b+\frac{1}{2}\right)_{n}}{n !\left(\frac{3}{2}-b\right)_{n}} c_{1} .
$$

A second solution of (3.1) is therefore given by

$$
y_{2}=c_{0} x^{-2 b}\left\{{ }_{2} F_{1}\left[\begin{array}{c}
a-2 b, a-b+\frac{1}{2} \\
\frac{1}{2}-b
\end{array} ; x^{2}\right]\right.
$$




$$
\left.-\frac{2(a-2 b) x}{1-2 b}{ }_{2} F_{1}\left[\begin{array}{c}
a-2 b+1, a-b+\frac{1}{2} ; x^{2} \\
\frac{3}{2}-b
\end{array}\right]\right\}
$$

when $|x|<1$.

It then follows, when $|x|<1$ and $\left|4 x /(1+x)^{2}\right|<1$ and provided $2 b+1$ is neither zero nor a negative integer, that there exist constants $A$ and $B$ such that

$$
(1+x)_{2}^{-2 a} F_{1}\left[\begin{array}{c}
a, b \\
2 b+1
\end{array} ; \frac{4 x}{(1+x)^{2}}\right]=A y_{1}+B y_{2}
$$

Now the left-hand side of (3.4) and the solution $y_{1}$ are both analytic at $x=0$, whereas the solution $y_{2}$ is not analytic at $x=0$ due to the presence of the factor $x^{-2 b}$. Hence $B=0$ and, by putting $x=0$ in (3.4), it is easily seen that $A=1$. If $2 b+1$ is a positive integer, the second solution may involve a logarithmic term; this again results in $B=0$. This then yields the result stated in (1.2).

A similar procedure can be employed to establish the quadratic transformation in (1.3). Putting $c=2 b-1$ in (2.1) and carrying out the same sequence of transformations, we obtain the differential equation satisfied by

$$
y=(1+x)^{-2 a} 2 F_{1}\left[\begin{array}{c}
a, b \\
2 b-1
\end{array} ; \frac{4}{(1+x)^{2}}\right]
$$

in the form

$$
x\left(1-x^{2}\right) \frac{d^{2} y}{d x^{2}}+\left\{2 b-1-2 x-(4 a-2 b+3) x^{2}\right\} \frac{d y}{d x}-2 a\{1+2(a-b+1) x\} y=0 .
$$

Substitution of (3.2) then leads to the three-term recurrence for the coefficients $c_{n}$

$$
\begin{aligned}
c_{1} & =\frac{-2(\lambda+a)}{(1+\lambda)(\lambda+2 b-1)} c_{0}, \\
c_{n} & =\frac{\{n+\lambda+2(a-1)\}\{n+\lambda+2(a-b)\} c_{n-2}+2(n+\lambda+a-1) c_{n-1}}{(n+\lambda)(n+\lambda+2 b-2)}
\end{aligned}
$$

for $n \geq 2$, subject to the indicial equation $\lambda(\lambda+2 b-2)=0$. The choice of indicial exponent $\lambda=0$ yields with the help of Mathematica the values of the coefficients given by

$$
c_{2 n}=\frac{(a)_{n}\left(a-b+\frac{3}{2}\right)_{n}}{n !\left(b-\frac{1}{2}\right)_{n}} c_{0}, \quad c_{2 n+1}=\frac{(a+1)_{n}\left(a-b+\frac{3}{2}\right)_{n}}{n !\left(b+\frac{1}{2}\right)_{n}} c_{1},
$$

with $c_{1}=2 a c_{0} /(2 b-1)$, and the choice $\lambda=2-2 b$ yields

$$
c_{2 n}=\frac{(a-b+2)_{n}\left(a-b+\frac{3}{2}\right)_{n}}{n !\left(\frac{3}{2}-b\right)_{n}} c_{0}, \quad c_{2 n+1}=\frac{(a-2 b+3)_{n}\left(a-b+\frac{3}{2}\right)_{n}}{n !\left(\frac{5}{2}-b\right)_{n}} c_{1},
$$


with $c_{1}=2(a-2 b+2) c_{0} /(3-2 b)$.

Consequently two solutions of the differential equation (3.6) are

$$
y_{1}=c_{0}\left\{{ }_{2} F_{1}\left[\begin{array}{c}
a, a-b+\frac{3}{2} \\
b-\frac{1}{2}
\end{array} x^{2}\right]+\frac{2 a x}{2 b-1}{ }_{2} F_{1}\left[\begin{array}{c}
a+1, a-b+\frac{3}{2} \\
b+\frac{1}{2}
\end{array}\right]\right\}
$$

and

$$
\begin{aligned}
y_{2}=c_{0} x^{2-2 b}\left\{{ }_{2} F_{1}\left[\begin{array}{c}
a-b+2, a-b+\frac{3}{2} \\
\frac{3}{2}-b
\end{array} x^{2}\right]\right. \\
\\
\left.\quad+\frac{2(a-2 b+2) x}{3-2 b}{ }_{2} F_{1}\left[\begin{array}{c}
a-2 b+3, a-b+\frac{3}{2} ; x^{2} \\
\frac{5}{2}-b
\end{array}\right]\right\}
\end{aligned}
$$

when $|x|<1$. It then follows, when $|x|<1,\left|4 x /(1+x)^{2}\right|<1$ and provided $2 b-1$ is neither zero nor a negative integer, that there exist constants $A$ and $B$ such that the function in (3.5) can be expressed as $A y_{1}+B y_{2}$. For the same reasons as in the previous case we find $A=1$ and $B=0$, thereby establishing (1.3).

\section{References}

[1] G. E. Andrews, R. Askey and R. Roy, Special Functions, Cambridge University Press, Cambridge, 2000.

[2] W. N. Bailey, Products of generalized hypergeometric series, Proc. London Math. Soc. 28(2) (1928) 242-254.

[3] J. L. Lavoie, F. Grondin and A. K. Rathie, Generalizations of Watson's theorem on the sum of a ${ }_{3} F_{2}$, Indian J. Math. 34 (1992) 23-32.

[4] F. W. J. Olver, D. W. Lozier, R. F. Boisvert and C. W. Clark (eds.), NIST Handbook of Mathematical Functions, Cambridge University Press, Cambridge, 2010.

[5] E. D. Rainville, Special Functions, Macmillan, New York, 1960.

[6] A. K. Rathie and Y. S. Kim, On two results contiguous to a quadratic transformation formula due to Gauss, Far East J. Math. Sci. 3(1) (2001) $51-58$.

Received: January 8, 2015; Published: January 29, 2015 